\title{
Opioid Prescriptions for New Low Back Pain: Trends and Variability by State
}

\author{
Micheal Raad, MD, Jina Pakpoor, MD, Andrew B. Harris, BS, \\ Varun Puvanesarajah, MD, Majd Marrache, MD, Joseph K. Canner, MHS, and \\ Amit Jain, $M D$
}

Background: The United States is facing a widespread opioid epidemic that disproportionately affects the working-age population. In the clinical setting, new low back pain is one of the most common reasons for opioid prescriptions, despite national recommendations advising against their use until nonopioid treatments have been trialed. In this study, we aimed to examine national opioid prescribing practices among primary care physicians after the evaluation of low back pain in working-age patients.

Method: This study used a national claims database's billing codes to identify patients in the outpatient setting with a new encounter for isolated low back pain following a 1-year look-back period. The primary outcome was whether an opioid prescription was filled within 30 days after the encounter. Patients with a daily morphine milligram equivalence (MME/day) known to be associated with a higher risk of overdose were also analyzed.

Results: A total of 418,565 patients between January 1, 2011 and November 30, 2016 were included. The proportion of patients with filled opioid prescriptions declined significantly between 2011 and $2016(P<.01 ; 28.5 \%$ in $2011,27.6 \%$ in $2012,26.3 \%$ in $2013,25.5 \%$ in $2014,23.5 \%$ in 2015 , and $20.4 \%$ in 2016). Nationally, the proportion of patients with a filled opioid prescription varied significantly between states $(P<.01)$, ranging from $12.9 \%$ in Hawaii to $33.6 \%$ in Arkansas.

Discussion: We found that the overall frequency of opioid prescriptions for low back pain is decreasing nationally, which speaks favorably for future initiatives to change physician prescribing patterns. However, we identified that there is large variation in prescribing patterns among physicians in different states. (J Am Board Fam Med 2020;33:138-142.)

Keywords: Low Back Pain, Opioids, Outpatients, Physician's Practice Patterns, Prescriptions, Primary Care Physicians, Primary Health Care, Quality of Health Care.

The United States is facing a widespread opioid epidemic that disproportionately affects the working-age population. In fact, 1 in 5 deaths in young adults between 25 and 34 years of age are related to opioid use. ${ }^{1}$ In the clinical setting, new low back pain is one

This article was externally peer reviewed.

Submitted 17 July 2019; revised 22 October 2019; accepted 23 October 2019.

From Department of Orthopedic Surgery, Johns Hopkins Hospital, Baltimore, MD (MR, ABH, VP, MM, AJ); Department of Radiology, Hospital of the University of Pennsylvania, Philadelphia, PA (JP); Department of General Surgery, Johns Hopkins Hospital, Baltimore, MD (JKC).

MR and JP contributed equally to this work and should both be considered first author.

Funding: none.

Conflict of interest: none declared.

Corresponding author: Amit Jain, MD, Johns Hopkins Hospital, Baltimore, MD, 21287 (E-mail: amitjain@jhmi.edu). of the most common reasons for opioid prescriptions, despite national recommendations advising against their use until nonopioid treatments have been trialed. $^{2,3}$ There is a lack of demonstrable long-term benefit of opioids and randomized clinical trials have shown their use to be associated with worse or similar long-term pain outcomes, as well as a dose-dependent risk of harm, compared with nonopioid medication. ${ }^{4,5}$

In light of the current nationwide focus on opioid-related health-care policy and future directives, we aim to examine opioid prescribing practices among primary care physicians after the evaluation of new low back pain in working-age patients.

\section{Methods}

This study used the IBM Health MarketScan ${ }^{\circledR}$ Commercial Claims and Encounters Database, 
Table 1. Characteristics of the Study Population

\begin{tabular}{lr}
\hline Characteristic & Value \\
\hline Study population (n) & 418,565 \\
Sex, female (\%) & 52.7 \\
Employee relation (\%) & \\
$\quad$ Employee & 65.2 \\
Spouse & 27.1 \\
Child/other & 7.8 \\
Region (\%) & \\
North East & 14.7 \\
North Central & 22.3 \\
South & 47.2 \\
West & 15.8 \\
Rural (\%) & 14.6 \\
Plain film radiograph* (\%) & 14.9 \\
\hline
\end{tabular}

*Proportion of the study population that had a plain film radiograph associated with the reference encounter for low back pain.

which contains health information on private-payer insurance beneficiaries across the United States. ICD-9 724.2 or ICD-10 M54.4 billing codes were used to identify patients in the outpatient setting with a new encounter for isolated low back pain following a 1-year look-back period. Patients with any concurrent encounter ICD code and, therefore, also of any concurrent spinal pathology diag- nosis, were excluded. In addition, excluded were patients who within the prior 12 months had an encounter billed in the database for a visit with a subspecialist physician, for example, an orthopedic surgeon or chronic pain specialist; patients who had filled opioid prescriptions within 90-days before their encounter of interest for low back pain; and patients who were captured in the database to have required forms indicative of chronic use.

The primary outcome was whether an opioid prescription of $\geq 20$ daily morphine milligram equivalence (MME) was filled within 30 days after the encounter. This dose was selected, as opioid doses of greater than $20 \mathrm{MME} /$ day have been shown to be associated with a higher risk of unintentional overdose compared with 0 to $20 \mathrm{MME} /$ day. ${ }^{3,5}$ Proportions were imputed with IBM Health MarketScan trends and sampling weights. The Cochran-Armitage trends test was used for trends analysis. State variation was analyzed using $\chi^{2}$ test.

\section{Results}

A total of 418,565 patients between January 1, 2011 and November 30, 2016 were included. Study population characteristics are displayed in Table 1 . In our study population, $25.7 \%$ [95\%

Figure 1. A caterpillar plot of the proportion of patients with a filled opioid prescription and the associated $95 \%$ logit confidence interval by state.

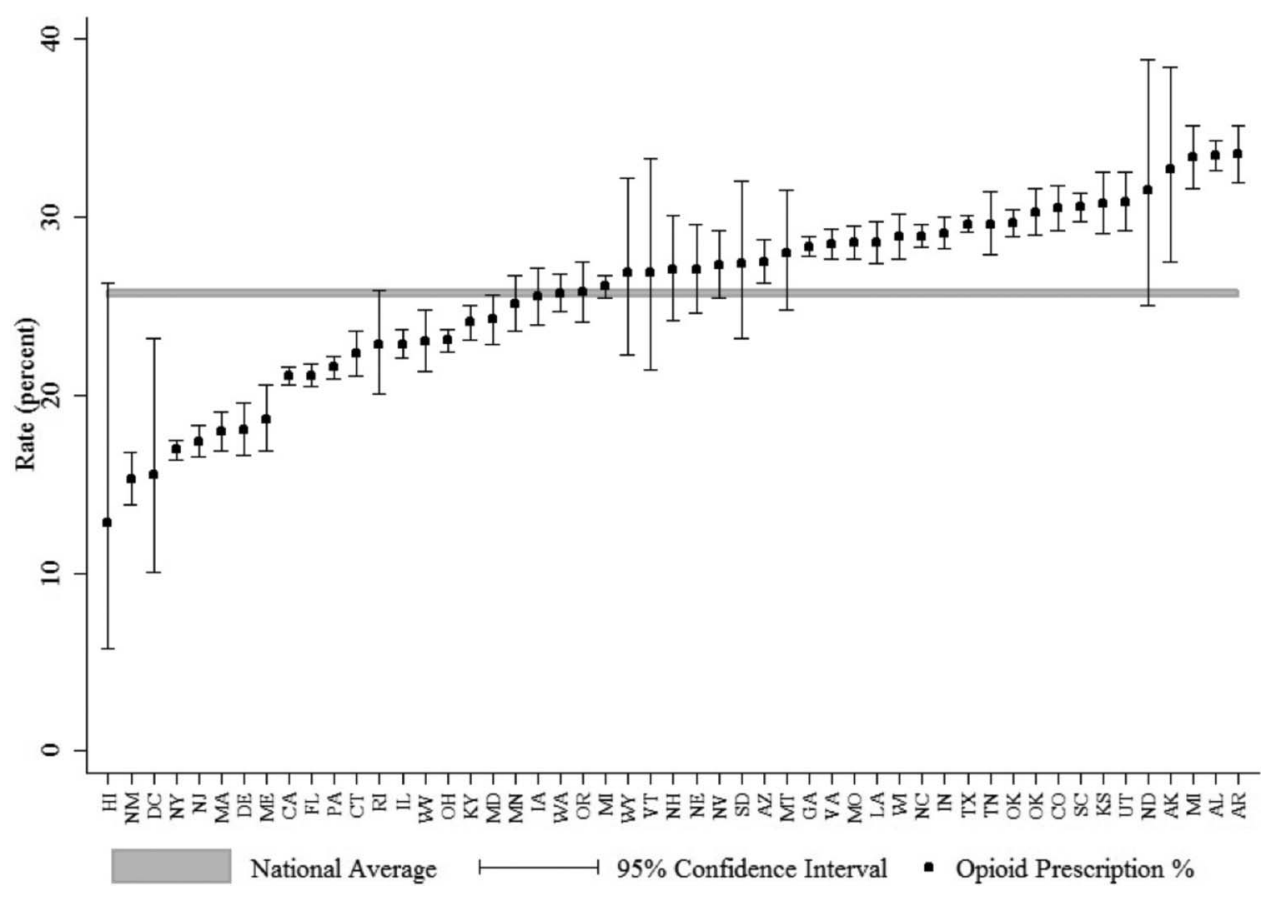


Figure 2. Regional scatter plots of the annual proportions of patients with any filled opioid prescription as well as the proportion of those with a filled prescription receiving more than $20 \mathrm{MME} /$ day and their associated $95 \%$ logit confidence intervals. a: Northeast. b: Midwest. c: South. d: West.
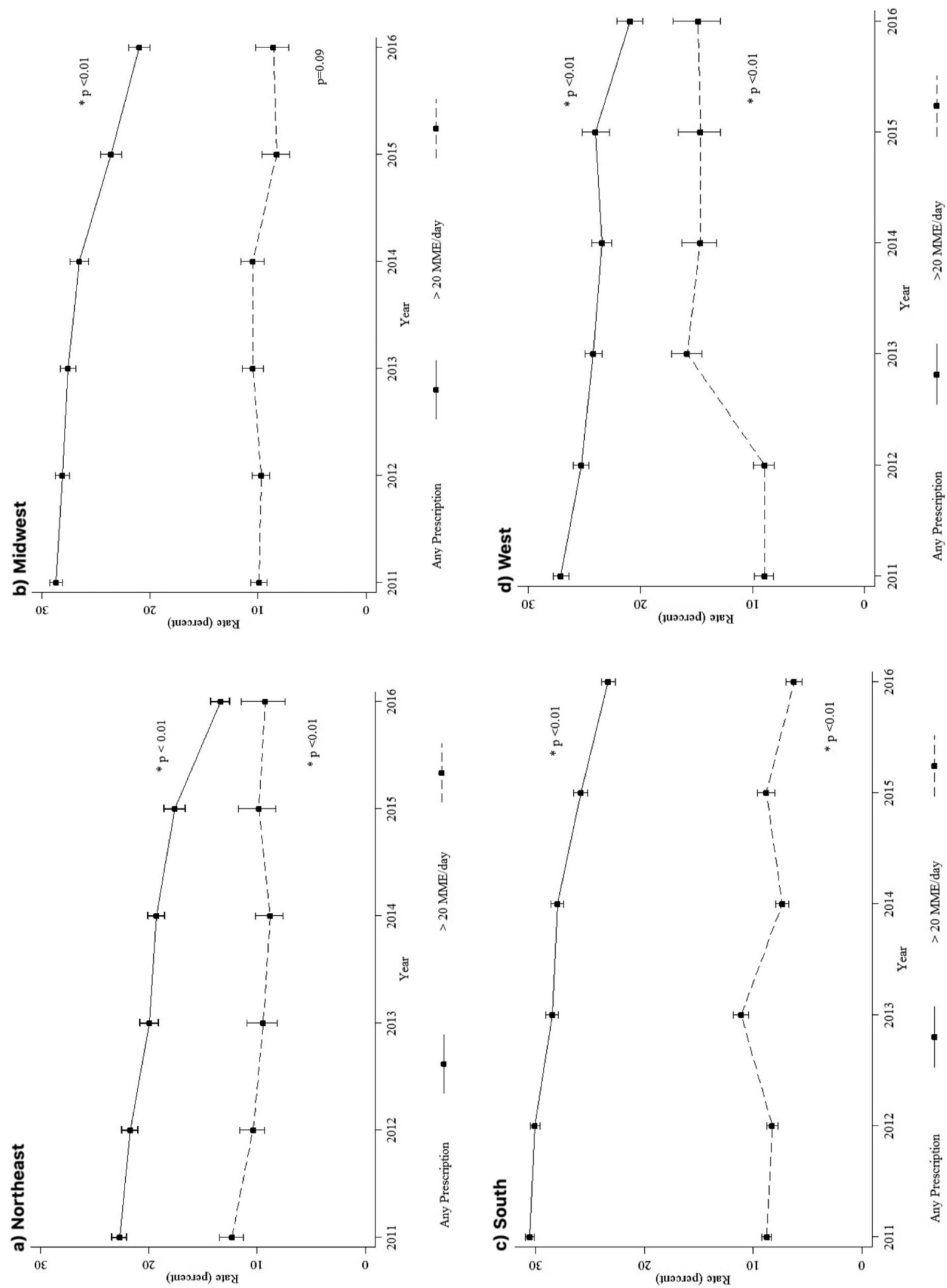
CI: 25.6-25.9] of patients had a filled opioid prescription 30-days postencounter. Nationally, the proportion of patients with a filled opioid prescription varied significantly between the various states $(P<.01)$, ranging from $12.9 \%$ in Hawaii to $33.6 \%$ in Arkansas (Figure 1). The proportion of patients with filled opioid prescriptions declined significantly between 2011 and $2016(P<.01 ; 28.5 \%$ in $2011,27.6 \%$ in 2012 , $26.3 \%$ in $2013,25.5 \%$ in $2014,23.5 \%$ in 2015 , and $20.4 \%$ in 2016). This decline was evident across the Northeast, Midwest, South, and West (Figure 2). Of those with a filled opioid prescription, the proportion with $>20 \mathrm{MME} /$ day did not significantly change over time $(P=.16)$.

\section{Discussion}

Encouragingly, the overall frequency of opioid prescriptions for new back pain encounters is decreasing nationally, which speaks favorably for future initiatives to change physician prescribing patterns. The underlying reasons for the reduction in opioid prescribing is unclear but almost certainly multifactorial. Such factors likely include an increased awareness for the current opioid epidemic in the United States and increased focus in national educational campaigns. For example, the American Board of Internal Medicine foundation's Choosing Wisely campaign has disseminated recommendations against the use of opioids for back pain before the trial of alternatives. ${ }^{6}$ These recommendations are supported by the American Association of Family Physicians. Other strategies may include increased use of state-wide prescription drug monitoring programs and "Harm Reduction" strategies. The latter strategy aims to decrease death from overdose through a combination of opioid substitutions, opioid education, concurrent supply of an opioid antagonist, and access to behavioral health services. ${ }^{7,8,9}$

However, although the overall trend is favorable, we further found that there is large geographic variation in prescribing patterns among physicians in different states. These findings add evidence to a current knowledge base that there is large variation in opioid prescribing among ordering providers and low back pain is no exception. ${ }^{10,11,12}$ Addressing geographic variation and discrepancies through local health policies and education efforts will continue to be needed to address the epidemic further. In addition, opioid pre- scriptions written at doses of $\geq 20 \mathrm{MME}$ remained relatively stable in our study. In addition to current strategies, there may, therefore, be a need for clear unified guidelines not only for appropriate indications but also for dosing.

In addition to the role played by physicians in combating opioid overuse, there is also an opportunity for third-party payers in guiding high-value care, and recent evidence has demonstrated their utilization management requirements to be highly variable. ${ }^{13}$ Private insurers can restrict the reimbursement of inappropriate prescribing, for example, through prior authorization and visit requirements. Ultimately, opioid prescribing for low back pain continues to provide multiple challenges that will be best addressed by a multifaceted approach encompassing payers, physicians, and patients.

To see this article online, please go to: http://jabfm.org/content/ 33/1/138.full.

\section{References}

1. Gomes T, Tadrous M, Mamdani MM, Paterson JM, Juurlink DN. The burden of opioid-related mortality in the United States. JAMA Netw Open 2018;1: e180217.

2. Hurwitz EL, Randhawa K, Yu H, Cote P, Haldeman S. The Global Spine Care Initiative: a summary of the global burden of low back and neck pain studies. Eur Spine J 2018;27:796-801.

3. Dowell D, Haegerich TM, Chou R. CDC guideline for prescribing opioids for chronic pain-United States, 2016. MMWR Recomm Rep 2016;65:1-49.

4. Waljee JF, Brummett CM. Opioid prescribing for low back pain: what is the role of payers? JAMA Netw Open 2018;1:e180236.

5. Adewumi AD, Hollingworth SA, Maravilla JC, Connor JP, Alati R. Prescribed dose of opioids and overdose: a systematic review and meta-analysis of unintentional prescription opioid overdose. CNS Drugs 2018;32:101-16.

6. American Academy of Physical Medicine and Rehabilitation. Available from: http://www.choosingwisely. org/clinician-lists/aapmr-opiates-for-low-back-pain/. Published 2015. Accessed October 10, 2019.

7. Centers for Disease Control and Prevention. Promising state strategies. Available from: https://www. cdc.gov/drugoverdose/policy/index.html. Published Accessed October 10, 2019.

8. Barglow P. Commentary: the opioid overdose epidemic: evidence-based interventions. Am J Addict 2018;27:605-7.

9. Worley J. Managing opioid use in orthopaedic patients through harm reduction strategies. Orthop Nurs 2019;38:129-35. 
10. Delgado MK, Huang Y, Meisel Z, et al. National variation in opioid prescribing and risk of prolonged use for opioid-naive patients treated in the emergency department for ankle sprains. Ann Emerg Med 2018;72:389-400.e1.

11. McDonald DC, Carlson K, Izrael D. Geographic variation in opioid prescribing in the U.S. J Pain 2012;13:988-96.
12. Schieber LZ, Guy GP, Jr., Seth P, et al. Trends and patterns of geographic variation in opioid prescribing practices by state. United States, 2006-2017. JAMA Netw Open 2019;2:e190665.

13. Heyward J, Jones CM, Compton WM, et al. Coverage of nonpharmacologic treatments for low back pain among US public and private insurers. JAMA Netw Open 2018;1:e183044. 